\title{
Molecular dynamics simulations for genetic interpretation in protein coding regions: where we are, where to go and when
}

\author{
Juan J. Galano-Frutos, Helena García-Cebollada and Javier Sancho (
}

Corresponding author: Javier Sancho, Departamento de Bioquímica y Biología Molecular y Celular. Facultad de Ciencias. Universidad de Zaragoza. Pedro Cerbuna 12, Zaragoza 50009, Spain; Biocomputation and Complex Systems Physics Institute (BIFI), Joint Units BIFI-IQFR (CSIC) and GBs-CSIC, Zaragoza 50018, Spain; Aragon Health Research Institute (IIS Aragón), Zaragoza 50009, Spain; Tel.: (+34) 976 761286; Fax: (+34) 976 762123; E-mail: jsancho@unizar.es

\begin{abstract}
The increasing ease with which massive genetic information can be obtained from patients or healthy individuals has stimulated the development of interpretive bioinformatics tools as aids in clinical practice. Most such tools analyze evolutionary information and simple physical-chemical properties to predict whether replacement of one amino acid residue with another will be tolerated or cause disease. Those approaches achieve up to $80-85 \%$ accuracy as binary classifiers (neutral/pathogenic). As such accuracy is insufficient for medical decision to be based on, and it does not appear to be increasing, more precise methods, such as full-atom molecular dynamics (MD) simulations in explicit solvent, are also discussed. Then, to describe the goal of interpreting human genetic variations at large scale through MD simulations, we restrictively refer to all possible protein variants carrying single-amino-acid substitutions arising from single-nucleotide variations as the human variome. We calculate its size and develop a simple model that allows calculating the simulation time needed to have a 0.99 probability of observing unfolding events of any unstable variant. The knowledge of that time enables performing a binary classification of the variants (stable-potentially neutral/unstable-pathogenic). Our model indicates that the human variome cannot be simulated with present computing capabilities. However, if they continue to increase as per Moore's law, it could be simulated (at $65^{\circ} \mathrm{C}$ ) spending only 3 years in the task if we started in 2031 . The simulation of individual protein variomes is achievable in short times starting at present. International coordination seems appropriate to embark upon massive MD simulations of protein variants.
\end{abstract}

Key words: genetic interpretation; mutation-effect prediction tools; single amino acid variation; molecular dynamics simulation; protein stability; large-scale phenotype prediction

\section{Introduction}

Many diseases have a genetic component. The Online Mendelian Inheritance in Man (OMIM) database (https://www.omim.org/) reports so far (last update: 16 June 2019) 7129 phenotypes associated to genetic disorders in humans, which can be passed on from generation to generation. Over the last decades, an increasing number of disease-causing mutations have been identified thanks, in part, to efforts by international collaborative large-scale sequencing initiatives such as the Human Genome Project [1, 2], the Human Variome Project [3], the ENCODE Project [4], the HapMap Project [5] or the 1000 Genomes Project [6] among others, which have paved the way to more recent

Juan J. Galano-Frutos, $\mathrm{PhD}$, is a post-doctoral researcher working on molecular modeling, computational drug discovery and bioinformatics at the 'Protein Folding and Molecular Design (ProtMol)' group at BIFI, University of Zaragoza.

Helena García-Cebollada is a PhD fellow with a Degree in Biotechnology, member of the BIFI, working on bioinformatics issues at the 'Protein Folding and Molecular Design (ProtMol)' group.

Javier Sancho is a Biochemistry Professor. His laboratory investigates protein stability using experimental and computational approaches in order to improve rational protein stabilization strategies and existing genetic interpretation tools.

Submitted: 23 July 2019; Received (in revised form): 22 September 2019

() The Author(s) 2019. Published by Oxford University Press.

This is an Open Access article distributed under the terms of the Creative Commons Attribution Non-Commercial License (http://creativecommons.org/ licenses/by-nc/4.0/), which permits non-commercial re-use, distribution, and reproduction in any medium, provided the original work is properly cited. For commercial re-use, please contact journals.permissions@oup.com 
Table 1. Summary of up-to-date (16 June 2019) statistics for Homo sapiens provided by the main biological databases referred in this manuscript

\begin{tabular}{lll}
\hline Database (URL) & Feature & Entries or report \\
\hline Online Mendelian Inheritance in Man (OMIM) (https://www.omim.org) & Genetic disorders & 7129 \\
NCBI's dbSNP Short Genetic Variations Database (https://www.ncbi.nlm.nih.gov/snp) & SNPs & 605048595 \\
RCSB Protein Data Bank (https://www.rcsb.org/) & 3D protein structures & 1559 \\
Ensembl (Human GRCh38.p12) (https://www.ensembl.org/Homo_sapiens/Info/Annotation) & Protein-coding genes & 20465 \\
Human Gene Mutation Database (HGMD Professional 2019.1) (http://www.hgmd.cf.ac.uk/ac/ & Mutations & 256070 \\
index.php) & &
\end{tabular}

initiatives, e.g. the Cancer Genome [7] and the 100,000 Genomes [8] projects. According to the NCBI's dbSNP Short Genetic Variations database (build 151) [9], 1 billion uniquely mapped (non-redundant) human genetic variations have been reported, including single- and multiple-base variations, insertions and deletions (indels) and short tandem repeats. Advances in highthroughput sequencing techniques [10-12] have enabled wholeexome sequencing (WES) [13] and genome-wide association studies (GWAS) [14] on humans [15-17] and a number of model species [18-21], contributing to a better understanding of how single-nucleotide variations (SNVs), the most frequent variations present in DNA [22], are related to diseases. An accurate interpretation of SNVs constitutes a major challenge in genetics and health.

\section{Genetic variation, disease, protein structure and the proteome}

Genes contain the information required to produce proteins, the main components of the cellular machinery, combining 20 essential building blocks called amino acids. Changes in the amino acid sequence of proteins arise from variations in proteincoding regions of the DNA, including synonymous and nonsynonymous single-nucleotide variations (sSNVs and nsSNVs, respectively), as well as indels and multi-nucleotide variations (MNVs). Regarding their effects on individual phenotypes, frameshifts and non-sense SNVs are likely to produce null or totally dysfunctional proteins. The effects of missense nsSNVs, in-frame indel variations or MNVs are more difficult to assess. They may range from not affecting protein function to causing severe dysfunctionality of the encoded protein variants, but they can also enhance the original function of the protein [23, 24] or even drive the acquisition of a new one [25]. Missense and inframe indel variations may affect not only protein folding and the stability of the native protein conformation [26-28] but also protein expression [29], post-translational modification [30, 31] or binding affinity [32-34]. Typically, missense variations are described in a binary way as being deleterious/pathogenic/ damaging or tolerated/neutral/benign. In monogenic disorders, deleteriousness tends to be associated to loss of structural stability $[35,36]$ while impaired binding interactions with partners or cofactors $[34,37,38]$ might be frequent in more complex diseases where disease-associated variations may not affect protein stability so often [39].

As the three-dimensional structure of a protein determines its function, the availability of protein structures solved at atomic level can greatly facilitate understanding which mutations impact protein function and why [40-42]. Knowledge of the structure allows to perform a variety of in silico analyses, such as the calculation of structure-based properties [43, 44], the study of protein dynamics using methodologies such as molecular dynamics (MD) simulations [45] or the combined application of molecular docking [46] and MD approaches [47] to uncover functional issues related to protein/cofactor or protein/protein interactions. Advances in resolution of 3D protein structures over the last decades, including the efforts by international consortia [41, 48, 49], have gathered a wealth of structural information that has laid the foundation for proteome-wide structural analyses. To date (16 June 2019), 149518 structures including wild-type proteins, mutated variants, protein domains, protein/cofactor, protein/protein and protein/nucleic acids complexes are available in the Protein Data Bank (PDB) (https://www.rcsb.org/stats/growth/overall). Within this wealth of structural information, human proteins are the larger group. Still, those solved at atomic resolution (around 1559) constitute a small percentage of the estimated number of human genes ( 20400 , see Table 1$)$, which in turn represents a lower limit to the still unclear size of the human proteome [50,51].

\section{Human genetic variation space: the variome}

The combination of technological advances with GWAS has allowed large-scale identification of human variations ( $90 \%$ corresponding to SNV) (https://www.ncbi.nlm.nih.gov/snp/? term=human). A subset of them, responsible for human inherited disease, is collected in the Human Gene Mutation Database (http://www.hgmd.cf.ac.uk/ac/index.php) which, in its latest professional version as of June 2019, reports 256070 entries. Over $57 \%$ of these variations correspond to SNVs (missense/nonsense). Most probably, they only account for a small fraction of all clinically relevant genetic variants present in human genomes. Although mutations associated with complex diseases seem often to arise in non-coding regions [52], most well-annotated genetic diseases are linked to coding variants [53], which-fortunately-are the more amenable to structural analysis. Knowing the size of the human genetic variation space may be useful. If the functional implications of all possible human nsSNVs could be accurately calculated, the interpretation of those already reported and of those being found in the future would be greatly speeded up. In this respect, the number of nsSNVs that can exist, and therefore be found sooner or later in a given gene, can be computed easily. In previous work [54], we showed that a small 37-residue-long repeat of the LDL receptor could give rise to 227 nsSNVs, including many that had been related to familial hypercholesterolemia. The number of hypothetical nsSNVs for a given gene product can be calculated from the number of possible nsSNVs associated to each codon $\left(n S S N V c_{i}\right)$ and the absolute frequencies of codons in the secondary transcript (see Supporting Information Table S1). Likewise, the complete nsSNV space for all the human genes, human_nsSNVome (HumanV for short), can be accurately calculated from the $n S S N V c_{i}$ values together with the absolute frequencies of codons in coding regions, $\mathrm{fc}_{\mathrm{i}}$.

$$
\text { HumanV }=\sum_{i=1}^{61} f c_{i} * n s S N V c_{i}
$$


Those frequencies can be obtained from the HIVE online resource [55] (see Supporting Information Table S2) and the canonical protein sequences downloaded from UnitProt after a search filtered by 'reviewed:yes AND organism: "Homo sapiens (Human) [9606]"). A total of 66474822 nsSNVs are estimated this way, which will be referred to as the theoretical human nonsynonymous single nucleotide variome (t-human_nsSNVome; t-HumanV, for short) as opposed to the sub-space of it that has been already described, which will be referred to as the d-HumanV.

\section{Protein interaction space: the interactome}

Amino acid variations occurring at hotspots of protein binding interfaces can dramatically affect binding affinity. The STRING database [56, 57] includes 19257 human proteins for which protein-protein network connections have been reported (out of the $\sim 20400$ human genes reported so far). In humans, each protein participates in about 3-10 protein-protein interactions (PPIs) [58]. In this sense, in-depth characterization of protein-protein interaction networks (PPINs) is crucial to understanding cellular pathways and devising strategies to effectively treat human illnesses [59-61]. The complete set of PPI taking place in a defined biological context (organelle, cell, organism, etc.) constitutes its interactome. Large-scale PPI screening techniques, in particular the yeast two-hybrid method (Y2H) [62], have allowed to uncover complete interactomes in a number of species including humans [63]. Very recently, proteome co-evolutionary methods have also shown an impressive capacity of working with millions of protein pairs to systematically identify PPIs on the wholeproteome scale [64]. In this context, current estimations of the human interactome range from 130000 [65] to 650 000 PPI [66], excluding trans-organism PPI (relevant for infectious diseases).

A growing number of computational tools used for prediction of PPI and PPIN have been released over the last few years, e.g. iLoops [67] HOMCOS [68], COTH [69], InterPreTS [70] and PRISM [71]. Also, databases storing annotated and/or predicted PPI are currently available, e.g. DIP [72], BIND [73], PrePPI [74] and STRING [75]. One way to identify key residues involved in PPI, to then establish mutation/function relationships, is to focus on the identification of protein-protein interaction sites (PPISs). Prediction of PPIS is facilitated by recently developed computational resources such as predPPIS [76] and IntPred [77], as well as by many others previously released [78]. The algorithms implemented in PPI/PPIN and PPIS predictive tools utilize methods based on protein sequence data, structure data or a combination of these (hybrid approaches). Despite the analytical progress condensed in those applications, which use a variety of mathematical-statistical methods (e.g. support vector machine, random forest, neural network, Bayes, hidden Markov model), the accuracy achieved barely reach $80 \%$ in the best cases [67-71, 76-78]. This is likely due to the still very low number of structures of protein complexes available in the PDB. Therefore, the applicability of this type of predictive tools to clinical diagnosis and therapeutics is still limited.

\section{Approaches in use for the interpretation of mutation effects at the protein level}

Much of what we know about mutation-driven effects on protein stability, folding or protein-protein interactions has been revealed through mutational studies based on individual amino acid changes. Recently, deep mutational scanning (DMS), a high- scale DNA-sequencing-based method, is becoming an invaluable tool for experimental evaluation of missense variants [79-81]. DMS aims at testing in a single, multiplexed assay the effects of hundreds or thousands of variations by focusing on the presence of a target property (e.g. cell growth, presence of fluorescent reporter or ligand binding) in a large library of variants [79-81]. However, the proteome-wide DMS-based approach faces difficulties, such as the need for specific assays or the complexity of the equipment needed.

The interpretation of mutation effects at the protein level can also be approached in a predictive scenario using computational methods [22, 82]. Many computational tools have been developed over the last 20 years for the prediction of mutation effects (mutation-effect prediction tools, MEPT), some of which have become quite popular (e.g. SIFT [83-86], PolyPhen2 [87] or CADD [88], see Table 2).

As PPI/PPIN/PPIS prediction tools, most MEPT utilize methods based on evolutionary conservation of sequence (homology), structure and structure-derived data (structure-based) or a combination of the two (hybrid approaches). Some (metapredictors) combine outputs from several MEPTs to provide consensus scores (Figure 1 and Table 2). Representing each of these groups, SIFT (homology) is based on 'the degree of conservation of amino acid residues in sequence alignments derived from closely related sequences, collected through PSI-BLAST' [85]; HoTMuSiC (structure-based) 'uses standard and temperature-dependent statistical potentials combined with an artificial neural network to predict the change in melting temperature $\Delta \mathrm{T}_{\mathrm{m}}$ upon point mutations' [89]; Polyphen2 (hybrid) 'uses eight sequence-based and three structure-based predictive features which were selected automatically by an iterative greedy algorithm' [87]; and Meta-SNP (meta-predictor) provides a consensus score based on SNAP, SIFT, PhD-SNP and PANTHER outputs [90-92]. MEPTs based on structural data often try to calculate $\Delta \Delta G$, the change in free energy (i.e. in conformational stability) brought about by a single-amino-acid variation. For a comprehensive review on protein stability and folding principles, or for an explanation of the way to determine experimentally $\Delta \Delta G$, see references [93] and [94], respectively.

Although most existing MEPTs only provide predictions for single-amino-acid variants (SAVs), some recently developed applications, e.g. PROVEAN [95], are already able to generate predictions for multiple-amino-acid substitutions, insertions and deletions. While this progress is encouraging, and most published MEPTs are freely available, the maximum accuracy achieved by these methodologies so far ( 80-85\%) [96] strongly limits their usage in clinical diagnosis. One of the major caveats of current MEPTs' development is the limited size of the datasets available for training and validating these tools. In this context, DMS studies [79-81] and novel developments [97] are expected to provide MEPTs' community with validation and training sets larger than those currently available, which will boost the next generation of MEPTs.

\section{Protein dynamics in interpretation of mutations}

Perhaps due to the limited number of human protein structures available at the beginning of the MEPT era, most of these applications rely heavily on protein sequence data analysis (Table 2). Even MEPTs using hybrid approaches commonly include more sequence-based prediction features than structure-based ones. On top of that, many of the structure-based features selected as 


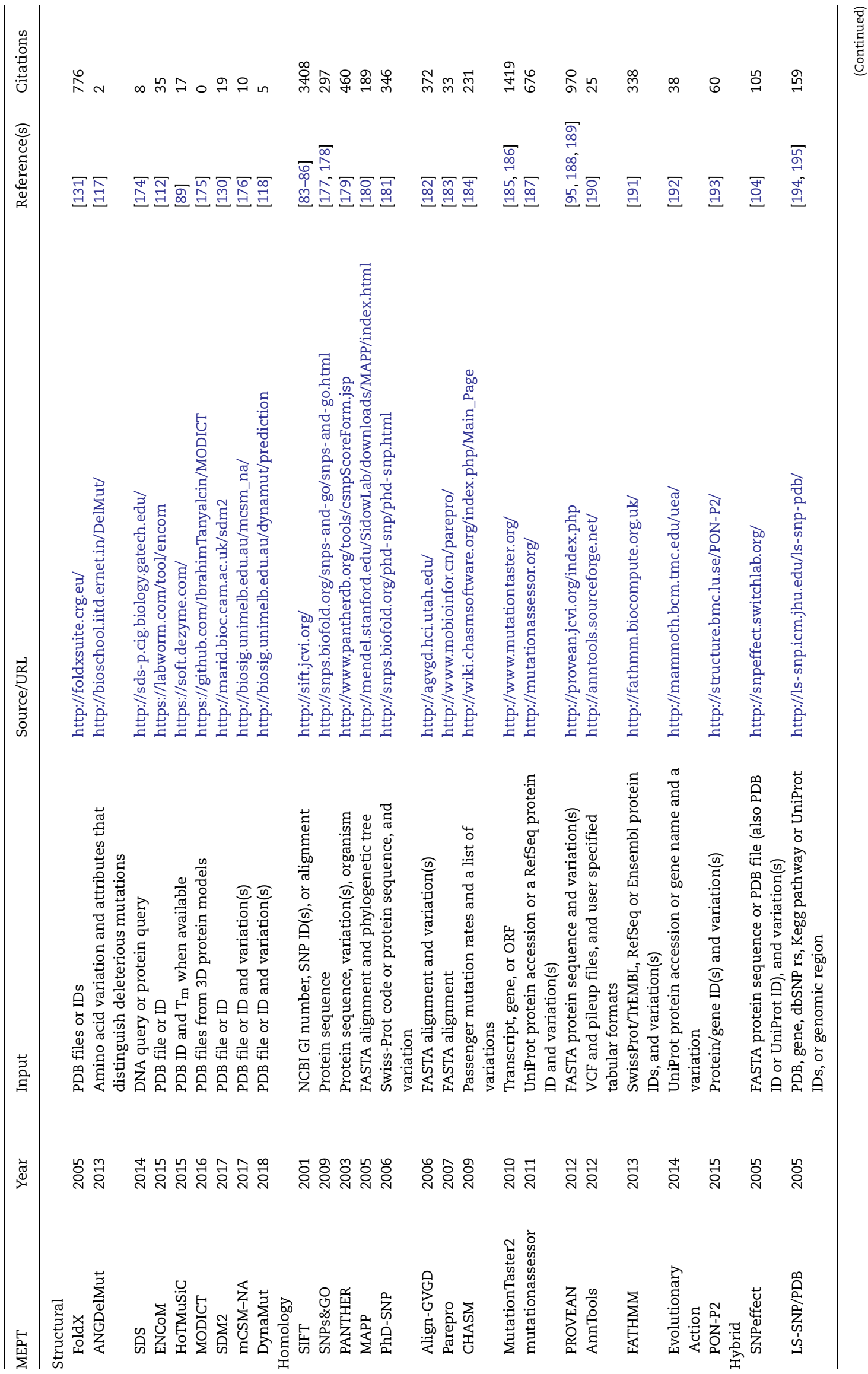




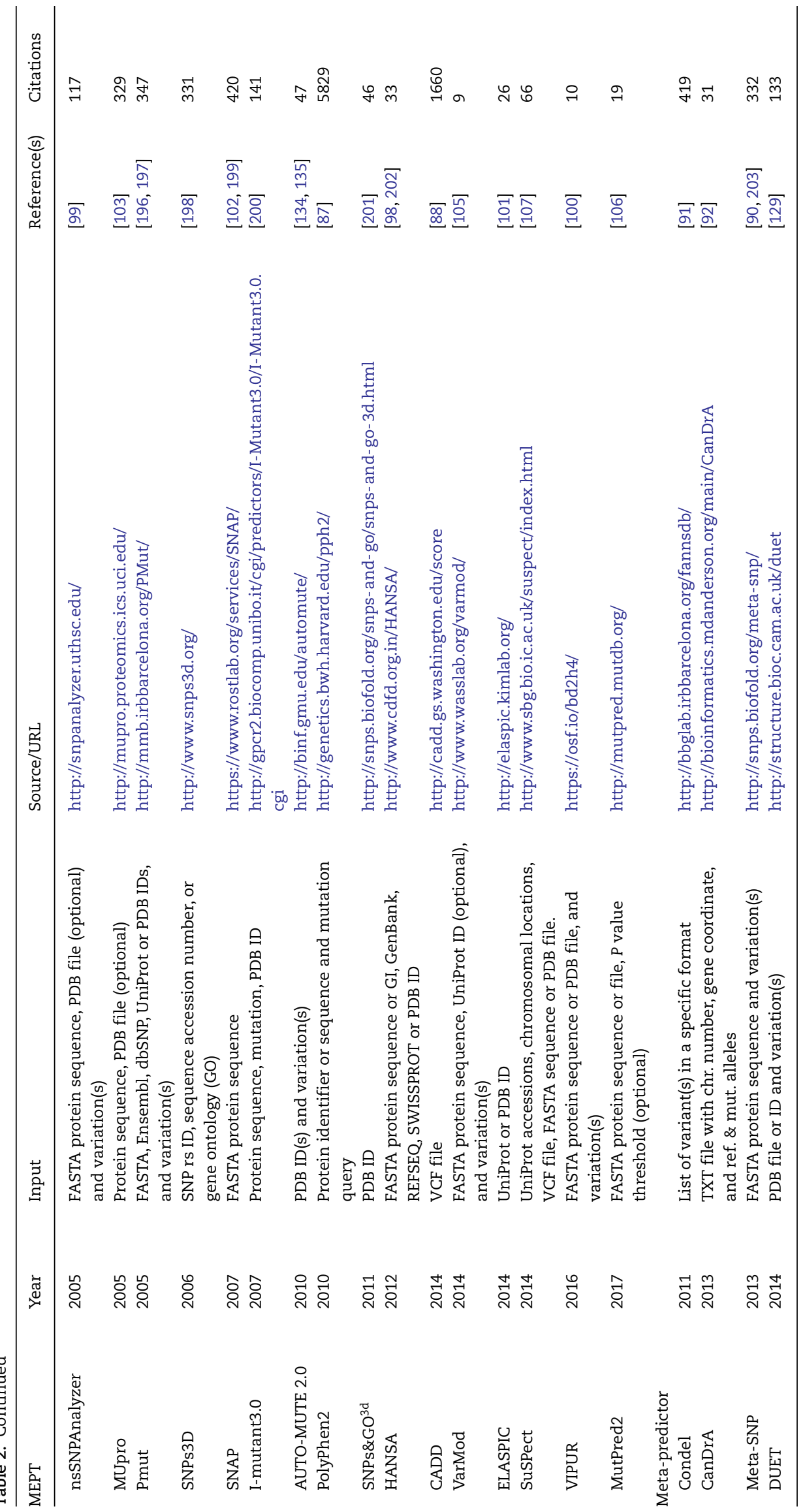


Input

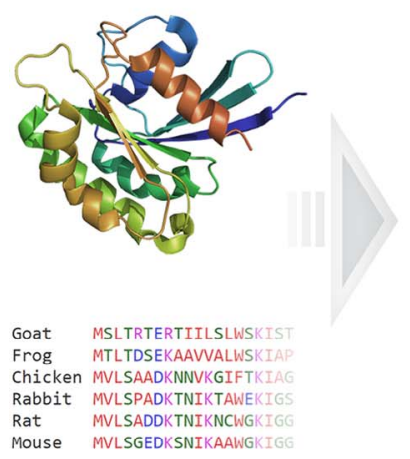

Algorithm

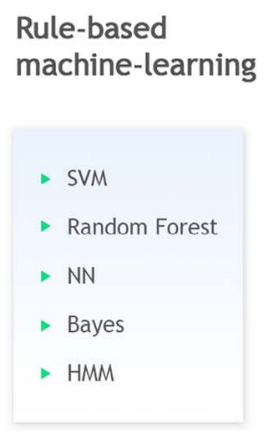

\section{Classification}

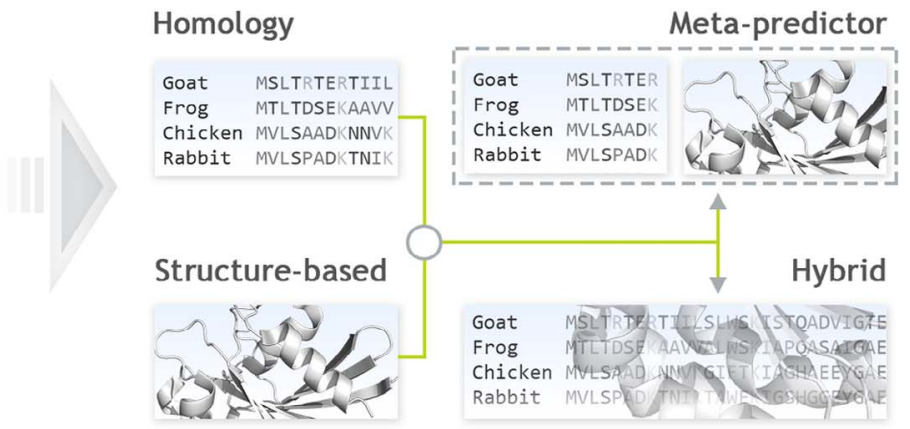

Figure 1. Overview of the methods underlying Mutation-Effects Prediction Tools (MEPTs). Homology methods rely on evolutionary conservation of protein sequence; structure-based ones utilize 3D structure or 3D-structure-derived data to train their algorithms (SVM: support vector machine; NN: neural networks; HMM: hidden Markov models); hybrid methods combine both sequence conservation and structural data; and meta-predictors obtain consensus scores based on outputs by individual MEPT.

predictors in MEPTs are obtained from either homology models or directly predicted from sequence rather than from true structural information [98-107]. This issue is being addressed in more recently developed MEPTs with the introduction of predictive features based on known 3D protein structures and on the usage of larger training sets including experimentally solved 3D protein structures. Nevertheless, such efforts have not led to significant increases in MEPTs' accuracies so far [108].

Looking for new approaches to enhance MEPTs' reliabilities, researchers have begun to exploit the availability of 3D protein structures to include dynamic aspects of proteins in the prediction of mutation effects [40, 47, 54, 109-120]. As protein function is defined by both structure and dynamics [121-123], dynamic patterns have begun to be recognized as descriptive of proteins [124, 125]. To our knowledge, DynaMut [118] and ENCoM [112] are the only available general (non protein-specific) MEPTs that include assessment of protein dynamics for predicting the impact of mutations on protein stability. Those two MEPTs apply normal mode analysis (NMA) simulations [126], a coarse-grained version in the case of ENCoM. According to the authors, considering destabilizing mutations alone, DynaMut comparably performs to methods such as mCSM-NA (the current version of mCSM [127]), I-mutant3.0 (the current version of I-mutant2.0 [128]) and DUET [129] and outperforms ENCoM [112] and SDM2 [130]. On the other hand, ENCoM's authors compared this tool to several existing methods, i.e. FoldX [131], HoTMusic (an improved version of PoPMusic [132] whose current last version is PoPMuSiC Sym [133]), AUTO-MUTE [134, 135] and I-mutant2.0 [128], and concluded that 'ENCoM proved to be the most self-consistent and least biased method' but 'not the best overall predictive method when considering both stabilizing and destabilizing mutations together' [112]. Overall, even though many available MEPTs may show overestimated accuracies and bias, and comparisons between them by authors are at times misleading, DynaMut and ENCoM do not seem to overcome the maximum performances obtained by the best predictor with which they are compared. One reason for this may be that, although NMA-based methods add dynamics elements to staticstructure-based methods, they do not sample sufficiently the conformational landscape. Also of note, the NMA simulations in those methods are performed in absence of water, disregarding fundamental solvation interactions.
MD simulation is a powerful, reliable tool used to study protein stability, dynamics or function $[136,137]$. Very recently, its usefulness in accurately calculating protein folding energetics has been shown [138]. The possibility offered by MD simulation to explore the conformational energy landscape of proteins in very realistic settings, including explicit solvent molecules and specific solution conditions (e.g. temperature, $\mathrm{pH}$, concentration, pressure), alongside the continuous progress made in related areas, such as the development of force fields [139], water models [140] and next-generation graphics processing units (GPUs) (high-performance computing), makes this tool a logical choice to address the analysis of SAV. In this context, two different situations can arise when MD is used to simulate protein mutation effects. In the infrequent case where both the native and mutant structures are known, unfolding simulations can be performed taking the corresponding structural models as the starting points. In the usual case where only the wildtype structure is available, a starting model of the mutant structure should be constructed by modeling the single-amino-acid substitution. Then, the evolution in time of the modeled mutant structure would be simulated by MD. We refer to this approach as relaxation molecular dynamics (rMD) simulations because they describe the relaxation of the protein native structure after the introduction of a mutation.

\section{Relaxation molecular dynamics}

Given the artifactual nature of the starting model, the pertinence of this approach may be arguable. Nevertheless, a number of rMD-based approaches have been used to study mutation effects on phenotypes in the last few years [47, 54, 110, 116, 117, 119]. Many of these works showed quite good correlations between their predictions and experimental data and, at the same time, allowed to extract meaningful insights underlying the mechanisms through which mutations impair protein function. In one of these works, a web-based tool, ANGDelmut, used for the prediction and analysis of functional loss mechanisms of deleterious mutations related to amyotrophic lateral sclerosis (ALS), has been made available (http://bioschool.iitd.ernet.in/DelMut/) [117]. This is-as far as we know-the only currently available tool for prediction of SAV effects relying on a classical atomistic MD approach. However, ANGDelmut is implemented only for the 
analysis of angiogenin, the target protein in ALS. Simulations performed in [117] use an implicit-solvent MD approach that is faster than explicit-solvent ones but may not describe in full the important solvation effects associated to the protein dynamics.

The promising results obtained in works where classical atomistic MD simulations with explicit water were performed $[47,54,110,116,119]$ reinforce the idea that using MD-based methods under realistic conditions allows to accurately model and reveal the complexity of the structural changes associated to point mutations in proteins. In previous work, we followed this approach to explore the complete t-HumanV of the low-density lipoprotein receptor (LDL-r) LA5 domain by performing all-atom rMD simulations in explicit solvent. The LA5 domain is a $37-$ residue repeat that plays a key role in the uptake of LDL particles from the blood plasma and in their release in the endosome. The LA5 domain concentrates the highest rate of mutations reported as disease-causing for familial hypercholesterolemia (FH) [54]. A stability analysis based on principal component analysis (PCA) of MD trajectories, combined with PPI information of the binding site [141, 142], allowed to satisfactorily predict the pathogenicity of 49 out of the $50 \mathrm{FH}$ mutations known by the time and to obtain a higher true positive rate than that provided by PMUT, Condel and PolyPhen2. The reliability of this approach raises hopes that using MD-based methodologies to address the prediction of SAV's deleteriousness will greatly contribute to obtain higher accuracies than those shown by currently available MEPT. Likewise, it demonstrates that performing integrative approaches capable of exploiting PPI information is a way to address the prediction of mutation effects in a reliable manner, which is required if prediction is to be applied to diagnosis. In the above example [54], short MD simulations (20 ns productive phase) sufficed to capture structural differences between wild-type-like and pathogenic mutant conformational ensembles and so to unveil the (un)compatibility of the different variants with the native conformation of the small, 37-residue LA5 domain. However, it is quite likely that the simulation time span required to observe structural disruption in rMD trajectories of larger proteins will be larger or may vary considerably depending on protein size [143], on the intrinsic dynamics determined by protein folds or on specific folding/unfolding mechanisms [125, 143-145]. Furthermore, the PCA-based analysis method used in the study of the LA5 domain may or may not be adequate for larger proteins.

\section{Problems and challenges to address}

The unavailability of MEPT integrating MD simulations onto SAVpredictive approaches is clearly linked to the high computational cost of the task. The still insufficient computing power and the lack of novel algorithms that could further accelerate full-atom MD simulations constitute a challenge for the expansion and generalization of their use for massive prediction of SAV's deleteriousness. Many approaches have been suggested or implemented in order to speed up atomistic MD simulations. Temperature or pressure raising [146-148], the inclusion of molecules of denaturing agents such as urea $[149,150]$ and the application of force $[151,152]$ are options often chosen in simulations thought for unfolding. From a more general perspective, the use of higher or mixed time-step algorithms [153], split GPU-CPU algorithms [154], force fields and MD programs optimized for GPU architectures [155], special-purpose hardware designed specifically for MD simulations (a prominent case being the Anton platform [156]), implicit solvent [157] or the incorporation of multiscale modeling algorithms [158-160] may also help. Such advances have not been systematically implemented in SAV-predictive approaches, and at present, the computational cost associated to using rMD to provide accuracy to genetic diagnostic in a variome-wide scale continues being too high. Yet, the increasing number of SNVs being identified and of proteins with known 3D structure available argues for the consideration of that goal as a worthy endeavor claiming for new concerted efforts.

There is also a challenge in performing quantitative analysis of MD trajectories in simulations of protein folding/unfolding events [161], specially in large-scale simulation projects. At present, a broad set of analyses intended for detecting protein conformational changes in MD trajectories are available, which often focus on basic root mean square deviations (RMSDs) and root mean square fluctuations (RMSFs) of atomic positions, gyration radius, secondary structure content, template modeling score (TM score), principal component analysis (PCA), percentage of native contacts, solvent-accessible surface area (SASA), hydrogen bonds number, phi and psi backbone angles, distances, etc. [162-164]. Albeit promising results have been obtained using these analysis methods, as in the aforementioned study on the LA5 domain [54], it is difficult to establish robust metrics to quantitatively assess conformational changes from trajectory analyses and, therefore, to accurately determine the impact of mutations on protein stability. In this context, there is a developing research field relying on the so-called higher-order statistics methods wherein the semi-automated analysis of large sets of data, e.g. from long MD simulations, is intended to efficiently and accurately detect conformational changes in molecular ensembles [165]. As this area is still in an early stage of development, a close collaboration between structural biologists, programmers and developers of MD tools is needed.

While protein structure prediction methods continue to improve [166], a high-resolution 3D all-atom protein structure remains the starting point of choice required to reliably perform full-atom MD simulations. In this sense, the current availability of 3D structures of human proteins still constitutes a limitation to the expansion of MD usage in SAV-predictive approaches. Related to this is the question of how many proteins constitute the human proteome and, therefore, how many simulations need to be done to analyze the complete tHumanV once their structures are solved. The answer is not trivial. Although the Human Genome Project revealed a lowerthan-anticipated number of genes, 20400 , a much higher number of protein species (proteoforms) can be produced in an individual from alternatively spliced RNA transcripts and from post-translational modifications [51, 167]. Besides, a variety of additional chemical entities including, for instance, cofactors or ions, as well as acetylated, phosphorylated and/or methylated residues, may appear in the scene, which increases the complexity of the task.

\section{Estimation of computational resources needed to perform rMD simulations on the complete HumanV at $25^{\circ} \mathrm{C}$}

Performing rMD for the whole t-HumanV, as it has been defined in a previous section, is a formidable task, and the time required with present and foreseeable computational capabilities should be estimated in order to decide when embarking upon the calculation would be appropriate. As explained above, the t-HumanV comprises $66474822 \mathrm{nsSNV}$. Thus, the real time $(\mathrm{T})$ required to simulate all the variants arising in the 20410 different proteins (canonical transcripts) and being able to provide an accurate 


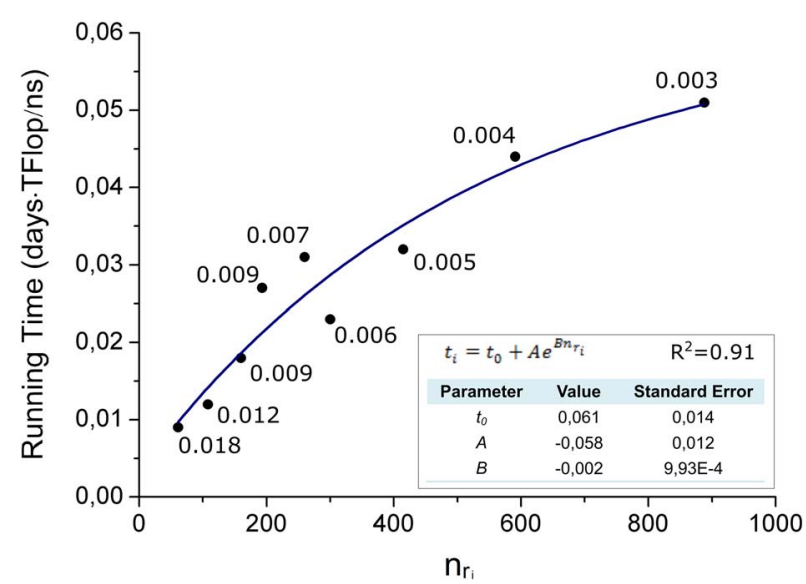

Figure 2. Running times (in days . TFlop/ns) versus number of residues $\left(n_{\mathrm{ri}}\right)$ fitting for nine proteins (see Table S3) simulated (three replicas of each protein) using the CHARMM27 force field, the TIP3P water model, an octahedron solvation box (with $1 \mathrm{~nm}$ minimum distance between protein surface and the end of the solvation box) and 48 Intel Xeon E5-2680v3 2.5GHz cores. Values indicated next to the points were calculated by dividing the radius of gyration of the protein by its number of residues (a structural sparseness measure, Table S3). These values decreasing with protein length leads to a lower number of water molecules per residue needed for the solvation box of larger proteins. The inset displays the fitting equation, the fitted parameters, their standard errors and the squared Pearson coefficient.

binary prediction can be expressed as

$$
\mathrm{T}=\sum_{i=1}^{20410} \sum_{j=1}^{n_{r_{i}}} n s S N V c_{j} \times \frac{t_{i}}{\text { TFlops }} \times \tau_{x_{\text {unf }}}
$$

where is the number of amino acid residues of protein $i, t_{i}$ is the running time required to simulate a unitary time span (e.g. $1 \mathrm{~ns}$ ) of protein $i$ using 1 TFlop of computing power, TFlops is the number of TFlops used in the calculation and $\tau_{x_{\text {unf }}}$ is the simulation time span (in ns) required to arrive to a given molar fraction $\left(x_{\mathrm{unf}}\right)$ of unfolded molecules (or to have a probability $x_{\mathrm{unf}}$ of observing unfolding in a single simulation, see below).

To obtain an estimation of the term $t_{i}$ in Equation 2, we have simulated nine roughly spherical globular proteins, containing from 60 to 900 residues (see Supporting Information Table S3). The simulations (300 ns long; three replicas of each protein) have been done using octahedral solvation boxes affording for a $1 \mathrm{~nm}$ minimum distance from protein surface atoms to the end of the solvation box, using the CHARMM27 force field, TIP3P waters and 48 Intel Xeon E5-2680v3 2.5GHz cores. Values of $t_{i}$ are processortype-independent as they are calculated for 1 TFlop (the number of TFlops is explicitly introduced in Equation 2). These values as a function of protein length (Supporting Information Table S3) are shown in Figure 2, fitted to an exponential function (Equation 3):

$$
t_{i}=t_{0}+A \times e^{B \times n_{r_{i}}}
$$

where $t_{0}, A$ and $B$ are fitting constants. The reasonably good fit of the experimental data to this simple function allows to estimate the time needed to simulate $1 \mathrm{~ns}$ for the different proteins of the proteome as: $t_{i}=0.061-0.058 e^{-0.002 n_{r_{i}}}$, although proteins with non-spherical global shapes may require longer times due to the increase in the number of water molecules conforming the solvation shell in the rMD simulations.

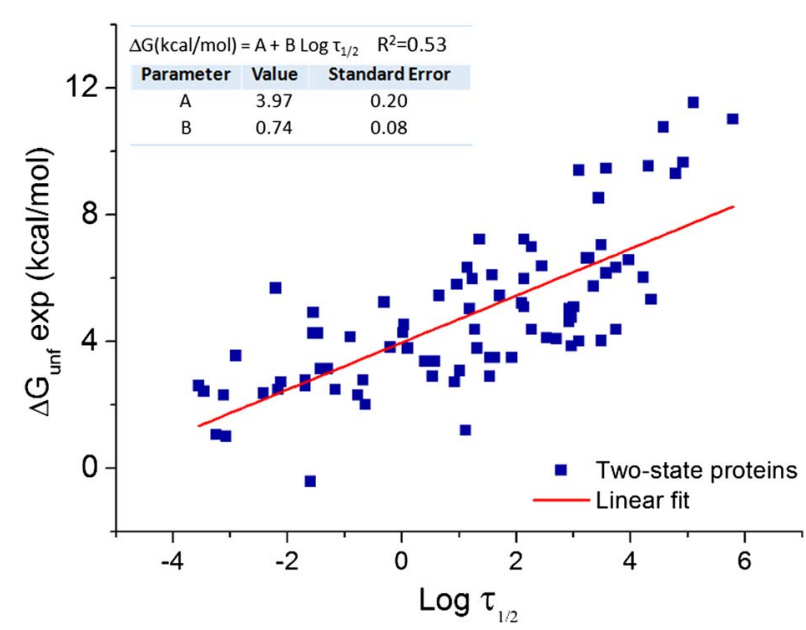

Figure 3. Correlation plot between protein conformational stability and the logarithm of unfolding half-lives showing a linear fit and the fitting parameters. The protein conformational stabilities have been calculated from folding and unfolding rate constants $\left(\Delta G_{\text {unf }}=-R T \times \ln \left(k_{\mathrm{u}} / k_{\mathrm{f}}\right)\right)$ of 89 two-state proteins (Manavalan et al. dataset) normalized at $25.0^{\circ} \mathrm{C}$. [168]. Half-lives have been calculated as $\tau_{0.5}=-\ln 2 / k_{\mathrm{u}}$. The inset displays the fitting equation, the fitted parameters, their standard errors and the squared Pearson coefficient.

When it comes to the term $\tau_{x_{\text {unf }}}$ in Equation 2, we have used normalized experimental data of folding $\left(k_{\mathrm{f}}\right)$ and unfolding $\left(k_{\mathrm{u}}\right)$ rate constants of 89 two-state proteins [168] to calculate their conformational stabilities $\left(\Delta G_{\text {unf }}\right)$ from the $\Delta G_{\text {unf }}=-\mathrm{RT} \times \ln$ $\left(k_{\mathrm{u}} / k_{\mathrm{f}}\right)$ relationship. Then, we have obtained an approximately linear correlation between the conformational stabilities of the protein and the logarithm of their half-lives of unfolding $\left(\tau_{0.5}\right)$ (Equation 4):

$$
\Delta G_{\mathrm{unf}}=3.97+0.74 \times \log \tau_{0.5}
$$

indicating that more stable proteins take longer to unfold (see Figure 3). If conformational stabilities directly determined by chemical denaturation using the linear extrapolation method are used instead for calculating the correlation, a similar equation yielding slightly shorter unfolding times can be obtained (Supporting Information Figure S1).

Furthermore, the time required $\left(\tau_{x_{\text {unf }}}\right)$ to obtain a given fraction $\left(x_{\mathrm{unf}}\right)$ of unfolded molecules starting from a population of fully folded proteins can be described as

$$
\tau_{x_{\mathrm{unf}}}=-\tau_{0.5} \times \ln \left(1-\mathrm{x}_{\mathrm{unf}}\right) / \ln 2
$$

The conformational stability of a protein determines the percentage of folded molecules at equilibrium. For stable proteins, the percentage is close to $100 \%$, and it decreases as the protein becomes less and less stable. The stability of most functional folded proteins studied ranges from 3 to $15 \mathrm{kcal} / \mathrm{mol}$ [169]. A protein with a low stability of $3 \mathrm{kcal} / \mathrm{mol}$ still has, at $25^{\circ} \mathrm{C}, 99.4 \%$ of the molecules folded at any time. According to Equation 4, the average $\tau_{0.5}$ for a protein in the high stability range ( $\left.15 \mathrm{kcal} / \mathrm{mol}\right)$ is of $25 \pm 7$ million years (Myr), while that for one in the low stability range $(3 \mathrm{kcal} / \mathrm{mol})$ is of only $50 \pm 14 \mathrm{~ms}$. A protein that, due to a SNV, becomes destabilized to the point of having its function compromised will likely exhibit a lower conformational stability [170]. If we take $2 \mathrm{kcal} / \mathrm{mol}$ as the stability threshold below which a protein is not stable enough to perform its cellular function (3\% of its molecules will be unfolded at $25^{\circ} \mathrm{C}$ at any moment), the time needed to observe the unfolding of individual 


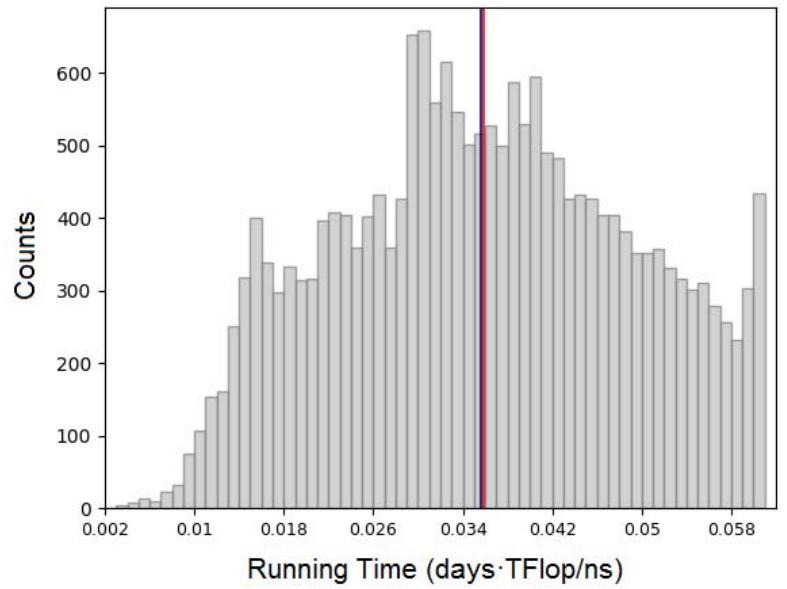

Figure 4. Histogram of estimated running times (in days.TFlop/ns) for all the proteins encoded in the human genome. The red line is the mean of the distribution (0.0357), and the navy blue line is the median (0.0358). The running time values were calculated with Equation 3 (see Figure 2).

protein molecules in experiment or simulation can be similarly calculated from Equation 4. As it turns out, half of the molecules of protein variants with stabilities of $2 \mathrm{kcal} / \mathrm{mol}$ or less will have experienced unfolding events in $2 \pm 0.6 \mathrm{~ms}$. Thus, if $2 \mathrm{~ms}$ of a single molecule of such destabilized protein variant are simulated at $25^{\circ} \mathrm{C}$, the probability of observing unfolding will be of just 0.5 . Equations 4 and 5 enable to calculate the average time needed for a given fraction of a protein of a given stability to become unfolded. Following with the example, 9 out of 10 molecules of that protein will be observed to unfold in $7 \pm 2 \mathrm{~ms}$ and, in $14 \pm 4 \mathrm{~ms}, 99 \%$ of the molecules will have unfolded. Therefore, if a single copy of the protein is simulated for $14 \mathrm{~ms}$, the probability of observing its unfolding will be of 0.99 . This $2 \mathrm{kcal} / \mathrm{mol}$ stability threshold is certainly arbitrary and should be only taken as a reasonable average value. This is so because protein variants causing amyloid-related diseases may become deleterious even if they display higher stabilities, as a small fraction of denatured molecules may initiate and drive the aggregation. On the other hand, proteins causing disease by a loss-of-function mechanism may provide some functionality to the cell even having stabilities below that level. It should be also borne in mind that some proteins display non-cooperative, i.e. non two-state, unfolding equilibria [94]. For those proteins, which may abound among the large ones, it is the unfolding of the weakest energetic domain [171] rather than that of the entire protein that should be monitored in the simulations. The weakest domain will correspond in most cases to the structural domain containing the deleterious variation, and it is the domain to which the $2 \mathrm{kcal} / \mathrm{mol}$ stability threshold applies.

Combining Equations 1, 2 and 3 and taking into account the length of the 20410 different proteins of the human proteome (which can be obtained from the canonical sequences annotated in UniProt, see Supporting Information Figure S2), the average time needed to simulate a single protein for $1 \mathrm{~ns}$ is $\bar{t}_{i}=0.0358$ days.TFlop (the distribution of $t_{i}$ values for the different proteins is shown in Figure 4). Thus, the approximate time needed to simulate 1 ns of the entire $\mathrm{t}$-HumanV is $731 \pm 306$ days.TFlop. This means that, in order to have a 0.99 probability of detecting any destabilized SAV with conformational stability below $2 \mathrm{kcal} / \mathrm{mol}, 29 \mathrm{Myr}$.TFlop would be required.
Differently to other MEPTs (e.g. FoldX, Rosetta or I-Mutant), the approach proposed here is based on the capabilities of MDtrajectory analysis to reliably detect conformational changes associated to very low conformational stability, Therefore, it does not allow, nor it tries, to compute $\Delta \Delta G$ values associated to mutations. However, recent developments in higher-order statistics methods to fine tune semi-automated analysis of long MD simulations [165] together with improved force fields [138] might allow in the future the quantitative analysis of conformational changes in MD trajectories, bringing an extra added value to MDbased predictive methods.

\section{When should we embark upon this endeavor?}

The very long time (29 Myr.TFlop) calculated above, which is beyond present computing capacity, is expected to decrease in the coming years both due to increased computing power of the CPUs and to improved efficiency of the MD-simulating algorithms. Taking a conservative approach, we may consider that the first factor will be the more relevant one and may accordingly disregard the improving contribution of the second factor. Then, assuming that the evolution of the computing power of CPUs will follow Moore's law [172], the time needed in the foreseeable future (at year $\mathrm{X}$ ) to compute $1 \mathrm{~ns}$ of the $\mathrm{t}$-HumanV becomes

$$
t^{@ \text { year } X}=\frac{t^{@ 2019}}{2^{(X-2019) / 2}}
$$

Combining Equations 2, 4 and 6 yields Equation 7 which allows to estimate the time required $\left(\mathrm{T}^{\mathrm{X}}\right)$ to simulate the complete human variome using an a priori defined computer power and beginning the simulations at year $\mathrm{X}$.

$$
\mathrm{T}^{\mathrm{X}}=\sum_{i=1}^{20410} \sum_{j=1}^{n_{r_{i}}} \frac{\mathrm{nsSNVc}_{j} \times\left(0.061-0.058 e^{-0.002 n_{r_{i}}}\right)}{\text { TFlops } \times 2^{(X-2019) / 2}} \times \tau_{x_{\mathrm{unf}}}
$$

If, as explained above, $\tau_{x_{\text {unf }}}$ is set as $14 \mathrm{~ms}$ (to have a 0.99 probability of observing the unfolding of protein variants with stability below $2 \mathrm{kcal} / \mathrm{mol}$ in any $\mathrm{rMD}$ simulation), we may estimate how long it would take to simulate the complete HumanV to identify any severely destabilizing mutation. Equation 7 indicates that beginning at present, 2019, and using the total TFlops allocated in the top 10 supercomputers in the TOP500 ranking (https://www.top500.org/) plus those accounted in the project of distributed computing Folding@Home (606826 TFlops, see Table 3), it would take around 193000 years to complete the simulations. However, if we began the simulation in 2051 , only $2.9 \pm 0.5$ years would be required using a similar number of supercomputers/projects of the time (see the blue curve in Figure 5A, and Table 3). Year 2051 would be the optimal moment to begin an efficient international effort to simulate $\mathrm{t}$-HumanV as it affords the earliest date of completion of the task (Figure 5A).

For the more modest task of calculating the entire variome of individual human proteins using the same stability threshold of $2 \mathrm{kcal} / \mathrm{mol}$, Figure 6 indicates that, starting at present, 2019, the mean time required to calculate a single protein variome is of 9.4 years, with half of the individual protein variomes needing less than 5.5 years.

When should we begin to simulate the human variome if we perform the simulations at $45^{\circ} \mathrm{C}$ or at $65^{\circ} \mathrm{C}$ ? 

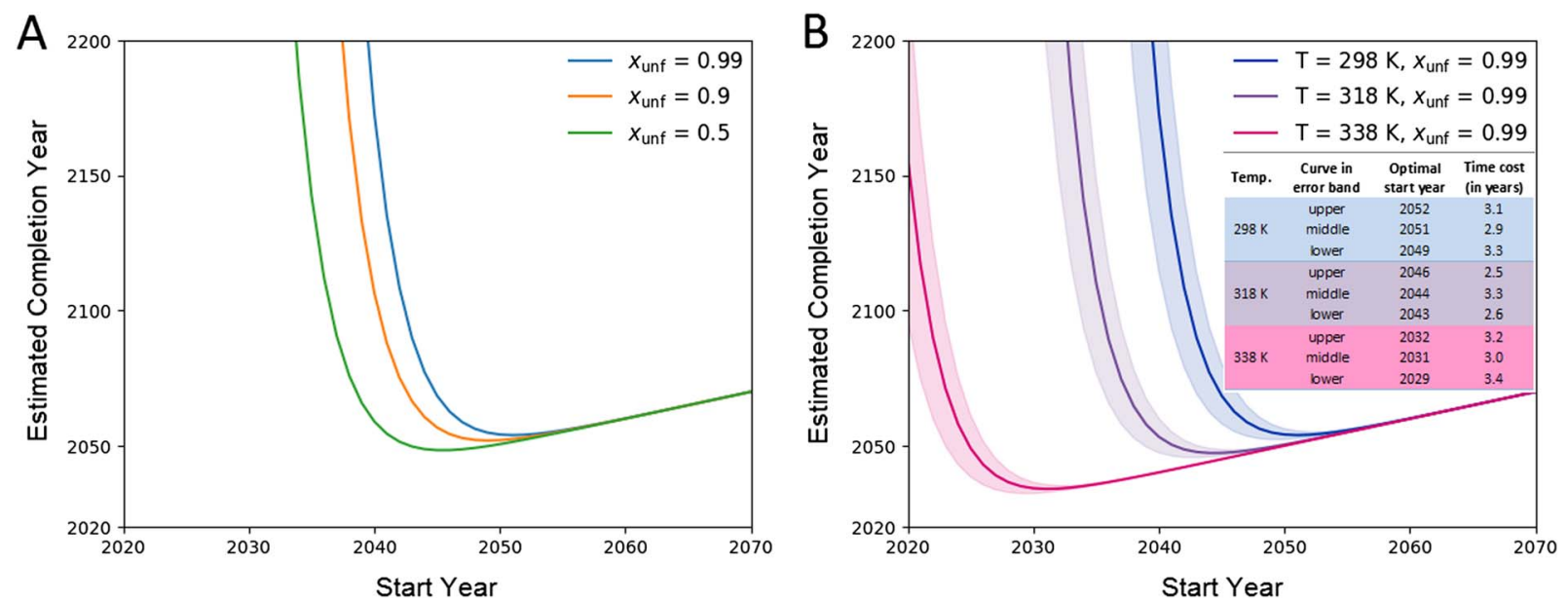

Figure 5. Estimated completion year versus start year profiles for the simulation of the entire t-HumanV. (A) Profiles calculated using different values of the fraction of unfolded molecules observed in the simulations (i.e. different probabilities of observing unfolding events in a single rMD simulation): $\mathrm{x}_{\text {unf }}$, a protein stability threshold of $2 \mathrm{kcal} / \mathrm{mol}$, and a simulation temperature of $25^{\circ} \mathrm{C}(298 \mathrm{~K})$. (B) Profiles calculated at three different temperatures by setting $\mathrm{x}_{\mathrm{unf}}=0.99$ and a protein stability threshold of $2 \mathrm{kcal} / \mathrm{mol}$. Depicted error bands have been calculated by propagating the fitting errors obtained for the different parameters (see the standard errors of the fittings in Figures 2 and 3) through Equation 7. The inserted table summarizes the optimal start years and time costs estimated for performing the required rMD simulations of the t-HumanV at the indicated temperatures. Profiles in both panels were calculated taking TFlops in Equation 7 as the number of TFlops allocated in the top 10 supercomputers worldwide plus those in the project of distributed computing Folding@Home (606 826, see Table 3) and their future equivalence according to Moore's law.

Table 3. Estimated time required to complete the simulation of the t-HumanV using the TFlops allocated in the Top Ten supercomputers worldwide plus those in the distributed computing project Folding@Home, or the expected TFlops in equivalent computing infrastructures in future years

\begin{tabular}{|c|c|c|c|c|}
\hline Supercomputer ranking & Supercomputer/project & Rmax (TFlop/s) & Start year ${ }^{\mathrm{a}}$ & Time required (years) ${ }^{\mathrm{a}}$ \\
\hline \multirow[t]{2}{*}{1} & Summit, DOE/SC/Oak Ridge National & 143500 & 2019 & 814757 \\
\hline & Laboratory, USA & & $2055^{\mathrm{b}}$ & 3.1 \\
\hline \multirow[t]{2}{*}{2} & Sierra, DOE/NNSA/LLNL, USA & 94640 & 2019 & 1235393 \\
\hline & & & $2056^{\mathrm{b}}$ & 3.3 \\
\hline \multirow[t]{2}{*}{3} & Sunway TaihuLight, National & 93015 & 2019 & 1902801 \\
\hline & Supercomputing Center in Wuxi, China & & $2056^{b}$ & 3.4 \\
\hline \multirow[t]{2}{*}{4} & Tianhe-2A, National Super Computer & 61445 & 2019 & 5507189 \\
\hline & Center in Guangzhou, China & & $2058^{b}$ & 2.6 \\
\hline \multirow[t]{2}{*}{5} & Piz Daint, Swiss National Supercomputing & 21230 & 2019 & 5799773 \\
\hline & Centre (CSCS), Switzerland & & $2061^{b}$ & 2.6 \\
\hline \multirow[t]{2}{*}{6} & Trinity, DOE/NNSA/LANL/SNL, USA & 20159 & 2019 & 5881167 \\
\hline & & & $2061^{b}$ & 2.8 \\
\hline \multirow[t]{2}{*}{7} & AI Bridging Cloud Infrastructure, AIST, & 19880 & 2019 & 6002856 \\
\hline & Japan & & $2061^{\mathrm{b}}$ & 2.8 \\
\hline \multirow[t]{2}{*}{8} & SuperMUC-NG, Leibniz Rechenzentrum, & 19477 & 2019 & 6646823 \\
\hline & Germany & & $2061^{b}$ & 2.9 \\
\hline \multirow[t]{2}{*}{9} & Titan, DOE/SC/Oak Ridge National & 17590 & 2019 & 6646823 \\
\hline & Laboratory, USA & & $2061^{b}$ & 3.2 \\
\hline \multirow[t]{2}{*}{10} & Sequoia, DOE/NNSA/LLNL, USA & 17173 & 2019 & 6808223 \\
\hline & & & $2061^{b}$ & 3.2 \\
\hline \multirow[t]{2}{*}{ Distributed computing project } & Folding@Home, The Pande Lab, Stanford & 98747 & 2019 & 1184012 \\
\hline & $\begin{array}{l}\text { University and Stanford University Medical } \\
\text { Center }\end{array}$ & & $2056^{\mathrm{b}}$ & 3.2 \\
\hline \multirow[t]{2}{*}{ - } & Top 10 + Folding@Home & 606826 & 2019 & 192671 \\
\hline & & & $2051^{\mathrm{b}}$ & 2.9 \\
\hline
\end{tabular}

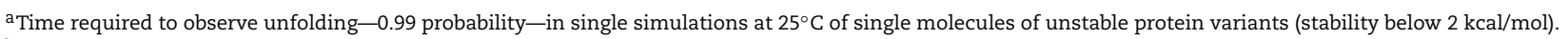
b Optimal moment-providing the earliest completion date-to begin the simulation of the complete human variome (see also Figure 5) using the corresponding TFlops calculated at that time as per Moore's law.

Rate constants of protein (un)folding are temperaturedependent. [173] The Eyring-Kramers equation

$$
k=\frac{k_{B} T}{h} \times e^{-\left(\frac{\Delta G^{\ddagger}}{R T}\right)}
$$

(where $k_{\mathrm{B}}$ and $h$ are Boltzmann's and Planck's constant, respectively) indicates that such dependency is modulated by the activation free-energy $\left(\Delta G^{\ddagger}\right)$, which can be determined experimentally. Analysis of experimental data on the variation of the unfolding rate constants of 11 proteins (ranging from 48 to 118 


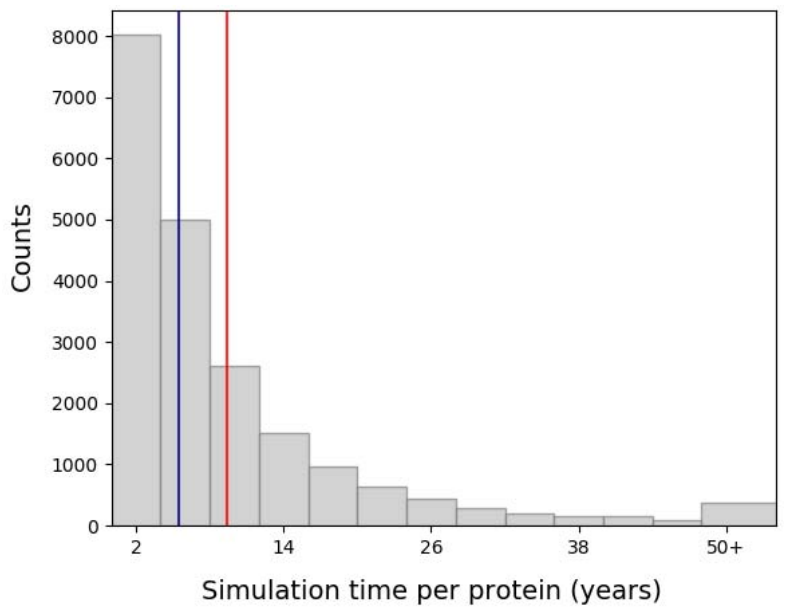

Figure 6. Histogram of the real simulation times (in years) required to simulate at present full individual protein variomes, calculated using Equation 7. The calculation was done by setting a protein stability threshold of $2 \mathrm{kcal} / \mathrm{mol}$ and a value of 0.99 for the probability of detecting that a particular SNV has lowered the stability of the wild-type protein below that threshold (i.e. setting $\tau_{\mathrm{x}_{\mathrm{unf}}}=14$ $\mathrm{ms})$, as well as a number of TFlops equivalent to those allocated together in the top 10 supercomputers and the distributed computing project Folding@Home at present (2019) (see Table 3). The red line represents the mean of the distribution (9.4), and the navy blue one, the median (5.5).

amino acid residues) as a function of temperature (Supporting Information Table S4) shows no correlation between the activation free energies of those proteins and their sizes (Supporting Information Figure S3). Therefore, we have calculated from that set of proteins an average activation free energy of unfolding of $82.5 \pm 12.1 \mathrm{~kJ} / \mathrm{mol}\left(\Delta G^{\ddagger} \pm \mathrm{SD}\right)$. Taking this value as an invariant, Equation 9 indicates that kinetic constants of protein unfolding $\left(k_{\mathrm{u}}\right)$ become 8.7 times larger if the temperature is raised from 298 to $318 \mathrm{~K}$, and 58.4 times larger if temperature is raised from 298 to $338 \mathrm{~K}$.

$$
\frac{k_{1}}{k_{2}}=\frac{T_{1}}{T_{2}} \times e^{\left(\frac{\Delta C^{\ddagger}}{R T}\right)\left(\frac{1}{T_{2}}-\frac{1}{T_{1}}\right)}
$$

As a consequence, the time needed to have a 0.99 probability of observing unfolding in a given $\mathrm{rMD}$ simulation performed at $298 \mathrm{~K}$, as described by Equations 4 and 5, is reduced at $318 \mathrm{~K}$ by a factor of 0.12 compared to that at $298 \mathrm{~K}$ and, at $338 \mathrm{~K}$, by a factor of 0.017 . As it seems, modest increases of simulation temperature can greatly reduce the computer time needed and bring the optimal moment when the simulation of the entire variome should be initiated significantly closer to present. The table inserted in Figure 5B summarizes the optimal start year for the simulation of the HumanV and the time cost at that point for the three simulation temperatures here discussed. While simulations at $25^{\circ} \mathrm{C}(298 \mathrm{~K})$ should begin in 2051 and last for 2.9 years, those at $45^{\circ} \mathrm{C}(318 \mathrm{~K})$ should begin in 2044 (lasting for 3.3 years), and those at $65^{\circ} \mathrm{C}(338 \mathrm{~K})$ should begin in 2031 and could be completed in 3.0 years.

As indicated above, the cost of simulating a single protein variome is much lower and, starting at present, 2019, the mean time required to simulate a single protein variome at $45^{\circ} \mathrm{C}$ would be of 1.2 years and, at $65^{\circ} \mathrm{C}$, of just 2 months. The simulation of individual protein variomes at temperatures close to the physiological one is already feasible, and that of the entire human variome will become feasible in few years. Having a reliable assessment of the structural impact of any single-amino-acid variation arising in the human proteome ready may greatly contribute to increase the accuracy of genetic interpretation of human SNV.

\section{Conclusions}

The continuous improvement of current MEPT based on evolutionary information and simple assessment of physicalchemical properties of amino acid residues appears not to be reaching the level of accuracy required for their generalized use in medical diagnosis. The exploration of alternative methods that fully analyze the impact of variations on protein structure, stability and binding, such as those using MD simulations, may provide the more accurate predictive tools in need. We have developed a simple model that allows to estimate the time needed to perform a predictive MD analysis of the entire human variome (here defined as all possible protein variants carrying a single-amino-acid replacement arising from a SNV in the corresponding coding sequence) with existing computing capabilities. Our model indicates that the structural impact of all human SAVs should begin to be assessed around 2031 and they could be completed by 2034 using explicit-solvent full-atom rMD simulations performed at a moderate temperature $\left(65^{\circ} \mathrm{C}\right)$. On the other hand, full variomes of individual proteins can be already analyzed at present, even at a lower temperature, closer to the physiological one.

\section{Supplementary Data}

Supplementary data are available online at https://academic. oup.com/bib.

\section{Author Contributions}

J.S. conceived and directed the investigation. J.J.G-F. and J.S. wrote the manuscript. J.J.G-F. and H.G-C. revised the literature, analyzed the data and made the figures and tables.

\section{Key Points}

- Obtaining genetic information describing variations in patients and healthy individuals has become easy and cheap, yet its accurate interpretation is still an unattained goal.

- Bioinformatics tools for genetic interpretation appear to have reached a maximum accuracy of around $85 \%$ in the binary interpretation of nsSNV in protein coding regions as deleterious or neutral, which is not sufficient for them to be trusted by clinicians.

- Full-atom molecular dynamics (MD) simulation of proteins carrying SAV offers good prospects of providing the extra accuracy needed, but this technique is slow, and its potential for massive interpretation of SAV at proteome scale needs to be appraised.

- We calculate here the size of the human nsSNV space (human Variome) at 66474822 protein variations and develop a physical model to determine the MD simulation time span required to have a high probability of observing unfolding of the unstable variants, a key datum enabling the use of MD simulations as binary predictors of protein stability.

- According to our model, an international effort initiated around 2031 could complete the MD simulation of the human Variome in 3 years, providing an accurate binary 
classification (destabilizing/not destabilizing) of all possible nsSNVs. For a single human protein of average size, it could be done at present in few months.

\section{Acknowledgements}

We thank the Biocomputation and Complex Systems Physics Institute (BIFI) of the University of Zaragoza for computing facilities granted to perform molecular dynamics simulations. We thank Alfonso López for his help in the creation of some figures.

\section{Funding}

This work was supported by the Ministerio de Economía y Competitividad, Spain (BFU2016-78232-P); Gobierno de Aragón, Spain (E45_17R); and ERDF-InterregV-A POCTEFA (PIREPRED-EFA086/15). The FPU16/04232 doctoral contract was conceded by the Ministerio de Educación, Cultura y Deporte, Spain, to H.G-C.

\section{References}

1. Shapiro LJ. Human genome project. West J Med 1993;158:181.

2. Hood L, Rowen L. The Human Genome Project: big science transforms biology and medicine. Genome Med 2013;5:79-9.

3. Cotton RG, Auerbach AD, Axton M, et al. The Human Variome Project. Science 2008;322:861-2.

4. Management EPS. The ENCODE (ENCyclopedia Of DNA Elements) Project. Science 2004;306:636-40.

5. Consortium IH. A second generation human haplotype map of over 3.1 million SNPs. Nature 2007;449:851-61.

6. Consortium GP, Abecasis GR, Altshuler D, et al. A map of human genome variation from population-scale sequencing. Nature 2010;467:1061-73.

7. Consortium TICG. International network of cancer genome projects. Nature 2010;464:993-8.

8. Peplow M. The 100000 Genomes Project. BMJ 2016;353: i1757.

9. Kitts A, Phan L, Ward M, et al. The Database of Short Genetic Variation (dbSNP) The NCBI Handbook [Internet], 2nd edn. Bethesda (MD): National Center for Biotechnology Information (US), 2013, Updated 2014 Apr 3.

10. Ambardar S, Gupta R, Trakroo D, et al. High throughput sequencing: an overview of sequencing chemistry. Indian $J$ Microbiol 2016;56:394-404

11. Park SJ, Saito-Adachi M, Komiyama Y, et al. Advances, practice, and clinical perspectives in high-throughput sequencing. Oral Dis 2016;22:353-64.

12. Reuter JA, Spacek DV, Snyder MP. High-throughput sequencing technologies. Mol Cell 2015;58:586-97.

13. Bick D, Dimmock D. Whole exome and whole genome sequencing. Curr Opin Pediatr 2011;23:594-600.

14. Witte JS. Genome-wide association studies and beyond. Annu Rev Public Health 2010;31:9-20.

15. Consortium WTCC. Genome-wide association study of 14,000 cases of seven common diseases and 3,000 shared controls. Nature 2007;447:661-78.

16. Gonzaga-Jauregui C, Lupski JR, Gibbs RA. Human genome sequencing in health and disease. Annu Rev Med 2012;63:35-61.
17. Yang Y, Muzny DM, Reid JG, et al. Clinical whole-exome sequencing for the diagnosis of mendelian disorders. $N$ Engl J Med 2013;369:1502-11.

18. Brachi B, Morris GP, Borevitz JO. Genome-wide association studies in plants: the missing heritability is in the field. Genome Biol 2011;12:232.

19. Horton MW, Bodenhausen N, Beilsmith K, et al. Genomewide association study of Arabidopsis thaliana leaf microbial community. Nat Commun 2014;5:5320.

20. Lee BY, Lee KN, Lee T, et al. Bovine genome-wide association study for genetic elements to resist the infection of footand-mouth disease in the field. Asian-Australas J Anim Sci 2015;28:166-70.

21. Wang K, Liu D, Hernandez-Sanchez J, et al. Genome wide association analysis reveals new production trait genes in a male Duroc population. PLos One 2015;10: e0139207.

22. Katsonis P, Koire A, Wilson SJ, et al. Single nucleotide variations: biological impact and theoretical interpretation. Protein Sci 2014;23:1650-66.

23. Blanco EH, Peinado JR, Martín MG, et al. Biochemical and cell biological properties of the human prohormone convertase 1/3 Ser357Gly mutation: a PC1/3 hypermorph. Endocrinology 2014;155:3434-47.

24. Isrie $M$, Breuss $M$, Tian $G$, et al. Mutations in either TUBB or MAPRE2 cause circumferential skin creases Kunze type. Am J Hum Genet 2015;97:790-800.

25. Tokuriki N, Stricher F, Serrano L, et al. How protein stability and new functions trade off. PLoS Comput Biol 2008; 4:e1000002.

26. Gaboriau DC, Rowling PJ, Morrison CG, et al. Protein stability versus function: effects of destabilizing missense mutations on BRCA1 DNA repair activity. Biochem J 2015; 466:613-24.

27. Sergouniotis PI, Barton SJ, Waller S, et al. The role of small in-frame insertions/deletions in inherited eye disorders and how structural modelling can help estimate their pathogenicity. Orphanet J Rare Dis 2016;11:125.

28. Shi Z, Sellers J, Moult J. Protein stability and in vivo concentration of missense mutations in phenylalanine hydroxylase. Proteins 2012;80:61-70.

29. Haraksingh RR, Snyder MP. Impacts of variation in the human genome on gene regulation. J Mol Biol 2013;425: 3970-7.

30. Reimand J, Bader GD. Systematic analysis of somatic mutations in phosphorylation signaling predicts novel cancer drivers. Mol Syst Biol 2013;9:637.

31. Yates CM, Sternberg MJ. The effects of non-synonymous single nucleotide polymorphisms (nsSNPs) on proteinprotein interactions. J Mol Biol 2013;425:3949-63.

32. Duning K, Wennmann DO, Bokemeyer A, et al. Common exonic missense variants in the $\mathrm{C} 2$ domain of the human KIBRA protein modify lipid binding and cognitive performance. J Translat Psych 2013;3:e272.

33. Feinberg H, Rowntree TJ, Tan SL, et al. Common polymorphisms in human langerin change specificity for glycan ligands. J Biol Chem 2013;288:36762-71.

34. Jubb HC, Pandurangan AP, Turner MA, et al. Mutations at protein-protein interfaces: small changes over big surfaces have large impacts on human health. Prog Biophys Mol Biol 2017;128:3-13.

35. Yue P, Li Z, Moult J. Loss of protein structure stability as a major causative factor in monogenic disease. J Mol Biol 2005;353:459-73. 
36. Wang Z, Moult J. SNPs, protein structure, and disease. Hum Mutat 2001;17:263-70.

37. David A, Razali R, Wass MN, et al. Protein-protein interaction sites are hot spots for disease-associated nonsynonymous SNPs. Hum Mutat 2012;33:359-63.

38. Nishi $\mathrm{H}$, Tyagi $\mathrm{M}$, Teng $\mathrm{S}$, et al. Cancer missense mutations alter binding properties of proteins and their interaction networks. PLos One 2013;8:e66273.

39. Sahni N, Yi S, Taipale M, et al. Widespread macromolecular interaction perturbations in human genetic disorders. Cell 2015;161:647-60.

40. Fleming N, Kinsella B, Ing C. Predicting protein thermostability upon mutation using molecular dynamics timeseries data. bioRxiv 2016;078246.

41. Montelione GT. The protein structure initiative: achievements and visions for the future. F1000 Biol Rep 2012;4:7.

42. Azia A, Uversky VN, Horovitz A, et al. The effects of mutations on protein function: a comparative study of three databases of mutations in humans. Israel Journal of Chemistry 2013;53:217-26.

43. Chwastyk M, Jaskolski M, Cieplak M. Structure-based analysis of thermodynamic and mechanical properties of cavity-containing proteins-case study of plant pathogenesis-related proteins of class 10. FEBS J 2014;281: 416-29.

44. Estrada J, Bernado P, Blackledge M, et al. ProtSA: a web application for calculating sequence specific protein solvent accessibilities in the unfolded ensemble. BMC Bioinformatics 2009;10:104.

45. Karplus M, Kuriyan J. Molecular dynamics and protein function. PNAS 2005;102:6679-85.

46. Barradas-Bautista D, Fernández-Recio J. Docking-based modeling of protein-protein interfaces for extensive structural and functional characterization of missense mutations. PLos One 2017;12:e0183643.

47. Priya Doss CG, Chakraborty C, Chen L, et al. Integrating in silico prediction methods, molecular docking, and molecular dynamics simulation to predict the impact of ALK missense mutations in structural perspective. Biomed Res Int 2014;895831.

48. Jones MM, Castle-Clarke S, Brooker D, et al. The structural genomics consortium: a knowledge platform for drug discovery: a summary. Rand Health Quarterly 2014;4:19.

49. Terwilliger TC, Stuart D, Yokoyama S. Lessons from structural genomics. Annu Rev Biophys 2009;38:371-83.

50. Kim MS, Pinto SM, Getnet D, et al. A draft map of the human proteome. Nature 2014;509:575-81.

51. Ponomarenko EA, Poverennaya EV, Ilgisonis EV, et al. The size of the human proteome: the width and depth. Int J Anal Chem 2016;2016:7436849.

52. Welter D, MacArthur J, Morales J, et al. The NHGRI GWAS Catalog, a curated resource of SNP-trait associations. Nucleic Acids Res 2014;42:D1001-6.

53. Ma M, Ru Y, Chuang L, et al. Disease-associated variants in different categories of disease located in distinct regulatory elements. BMC Genomics 2015;16:S3.

54. Angarica VE, Orozco M, Sancho J. Exploring the complete mutational space of the LDL receptor LA5 domain using molecular dynamics: linking SNPs with disease phenotypes in familial hypercholesterolemia. Hum Mol Genet 2016; 25:1233-46.

55. Athey J, Alexaki A, Osipova E, et al. A new and updated resource for codon usage tables. BMC Bioinformatics 2017; 18:391.
56. Szklarczyk D, Franceschini A, Wyder S, et al. STRING v10: protein-protein interaction networks, integrated over the tree of life. Nucleic Acids Res 2015;43:D447-52.

57. Szklarczyk D, Morris $\mathrm{JH}$, Cook $\mathrm{H}$, et al. The STRING database in 2017: quality-controlled protein-protein association networks, made broadly accessible. Nucleic Acids Res 2017;45:D362-8.

58. Futschik ME, Chaurasia G, Herzel H. Comparison of human protein-protein interaction maps. Bioinformatics 2007; 5:605-11.

59. Acuner Ozbabacan SE, Engin HB, Gursoy A, et al. Transient protein-protein interactions. Protein Eng Des Sel 2011; 24:635-48.

60. Ngounou Wetie AG, Sokolowska I, Woods AG, et al. Investigation of stable and transient protein-protein interactions: past, present, and future. Proteomics 2013;13:538-57.

61. Snider J, Kotlyar M, Saraon P, et al. Fundamentals of protein interaction network mapping. Mol Syst Biol 2015;11:848.

62. Brückner A, Polge C, Lentze N, et al. Yeast two-hybrid, a powerful tool for systems biology. Int J Mol Sci 2009;10:2763-88.

63. Rual JF, Venkatesan K, Hao T, et al. Towards a proteomescale map of the human protein-protein interaction network. Nature 2005;437:1173-8.

64. Cong Q, Anishchenko I, Ovchinnikov S, et al. Protein interaction networks revealed by proteome coevolution. Science 2019;365:185

65. Venkatesan K, Rual JF, Vazquez A, et al. An empirical framework for binary interactome mapping. Nat Methods 2009;6:83-90.

66. Stumpf MP, Thorne T, de Silva E, et al. Estimating the size of the human interactome. Proc Natl Acad Sci USA 2008; 105:6959-64.

67. Planas-Iglesias J, Marin-Lopez MA, Bonet J, et al. iLoops: a protein-protein interaction prediction server based on structural features. Bioinformatics 2013;29:60-2.

68. Fukuhara N, Kawabata T. HOMCOS: a server to predict interacting protein pairs and interacting sites by homology modeling of complex structures. Nucleic Acids Res 2008; $36: \mathrm{W} 185-9$.

69. Mukherjee S, Zhang Y. Protein-protein complex structure predictions by multimeric threading and template recombination. Structure 2011;19:955-66.

70. Aloy P, Russell RB. InterPreTS: protein interaction prediction through tertiary structure. Bioinformatics 2003;19:161-2.

71. Keskin O, Nussinov R, Gursoy A. PRISM: protein-protein interaction prediction by structural matching. Methods Mol Biol 2008;484:505-21.

72. Xenarios I, Rice DW, Salwinski L, et al. DIP: the database of interacting proteins. Nucleic Acids Res 2000;28:289-91.

73. Bader GD, Betel D, Hogue CW. BIND: the biomolecular interaction network database. Nucleic Acids Res 2003;31:248-50.

74. Zhang QC, Petrey D, Garzón JI, et al. PrePPI: a structureinformed database of protein-protein interactions. Nucleic Acids Res 2013;41:D828-33.

75. von Mering C, Huynen M, Jaeggi D, et al. STRING: a database of predicted functional associations between proteins. Nucleic Acids Res 2003;31:258-61.

76. Tzu-Hao K, Kuo-Bin L. Predicting protein-protein interaction sites using sequence descriptors and site propensity of neighboring amino acids. Int J Mol Sci 2016;17: 1788.

77. Northey TC, Barešić A, Martin ACR. IntPred: a structurebased predictor of protein-protein interaction sites. Bioinformatics 2018;34:223-9. 
78. Esmaielbeiki R, Krawczyk K, Knapp B, et al. Progress and challenges in predicting protein interfaces. Brief Bioinform 2016;17:117-31.

79. Fowler DM, Fields S. Deep mutational scanning: a new style of protein science. Nat Methods 2014;11:801-7.

80. Stein A, Fowler DM, Hartmann-Petersen R, et al. Biophysical and mechanistic models for disease-causing protein variants. Trends Biochem Sci 2019;44:575-88.

81. Fowler DM, Stephany JJ, Fields S. Measuring the activity of protein variants on a large scale using deep mutational scanning. Nat Protoc 2014;9:2267-84.

82. Potapov V, Cohen M, Schreiber G. Assessing computational methods for predicting protein stability upon mutation: good on average but not in the details. Protein Eng Des Sel 2009;22:553-60.

83. Kumar P, Henikoff S, Ng PC. Predicting the effects of coding non-synonymous variants on protein function using the SIFT algorithm. Nat Protoc 2009;4:1073-81.

84. Ng PC, Henikoff S. Predicting deleterious amino acid substitutions. Genome Res 2001;11:863-74.

85. Ng PC, Henikoff SSIFT. Predicting amino acid changes that affect protein function. Nucleic Acids Res 2003;31:3812-4.

86. Ng PC, Henikoff S. Predicting the effects of amino acid substitutions on protein function. Annu Rev Genomics Hum Genet 2006;7:61-80.

87. Adzhubei IA, Schmidt S, Peshkin L, et al. A method and server for predicting damaging missense mutations. Nat Methods 2010;7:248-9.

88. Kircher M, Witten DM, Jain P, et al. A general framework for estimating the relative pathogenicity of human genetic variants. Nat Genet 2014;43:310-5.

89. Pucci F, Bourgeas R, Rooman M. Predicting protein thermal stability changes upon point mutations using statistical potentials: introducing HoTMuSiC. Sci Rep 2016;6: 23257.

90. Capriotti E, Altman RB, Bromberg Y. Collective judgment predicts disease-associated single nucleotide variants. Mutations in proteins. BMC Genomics 2013;14:S2.

91. González-Pérez A, López-Bigas N. Improving the assessment of the outcome of nonsynonymous SNVs with a consensus deleteriousness score, Condel. Am J Hum Genet 2011; 88:440-9.

92. Mao Y, Chen $\mathrm{H}$, Liang $\mathrm{H}$, et al. CanDrA: cancer-specific driver missense mutation annotation with optimized features. PLos One 2013;8:e77945.

93. Dill KA, MacCallum JL. The protein-folding problem, 50 years on. Science 2012;338:1042.

94. Sancho J. The stability of 2-state, 3-state and more-state proteins from simple spectroscopic techniques... plus the structure of the equilibrium intermediates at the same time. Arch Biochem Biophys 2013;531:4-13.

95. Choi Y, Chan AP. PROVEAN web server: a tool to predict the functional effect of amino acid substitutions and indels. Bioinformatics 2015;31:2745-7.

96. Riera C, Padilla N, de la Cruz X. The complementarity between protein-specific and general pathogenicity predictors for amino acid substitutions. Hum Mutat 2016;37: 1013-24.

97. Nisthal A, Wang CY, Ary ML, et al. Protein stability engineering insights revealed by domain-wide comprehensive mutagenesis. Proc Natl Acad Sci 2019;116:16367.

98. Acharya V, Nagarajaram HA. Hansa: an automated method for discriminating disease and neutral human nsSNPs. Hum Mutat 2012;33:332-7.
99. Bao L, Zhou M, Cui Y. nsSNPAnalyzer: identifying diseaseassociated nonsynonymous single nucleotide polymorphisms. Nucleic Acids Res 2005;33:W480-2.

100. Baugh EH, Simmons-Edler R, Müller CL, et al. Robust classification of protein variation using structural modelling and large-scale data integration. Nucleic Acids Res 2016; 44:2501-13.

101. Berliner N, Teyra J, Colak R, et al. Combining structural modeling with ensemble machine learning to accurately predict protein fold stability and binding affinity effects upon mutation. PLos One 2014;9:e107353.

102. Bromberg Y, Rost B. SNAP: predict effect of nonsynonymous polymorphisms on function. Nucleic Acids Res 2007;35:3823-35.

103. Cheng J, Randall A, Baldi P. Prediction of protein stability changes for single-site mutations using support vector machines. Proteins 2006;62:1125-32.

104. De Baets G, Van Durme J, Reumers J, et al. SNPeffect 4.0: on-line prediction of molecular and structural effects of protein-coding variants. Nucleic Acids Res 2012;40:D935-9.

105. Pappalardo M, Wass MN. VarMod: modelling the functional effects of non-synonymous variants. Nucleic Acids Res 2014;42:W331-6.

106. Pejaver V, Urresti J, Lugo-Martinez J, et al. MutPred2: inferring the molecular and phenotypic impact of amino acid variants. bioRxiv 2017;134981.

107. Yates CM, Filippis I, Kelley LA, et al. SuSPect: enhanced prediction of single amino acid variant (SAV) phenotype using network features. J Mol Biol 2014;426:2692-701.

108. Grimm DG, Azencott CA, Aicheler F, et al. The evaluation of tools used to predict the impact of missense variants is hindered by two types of circularity. Hum Mutat 2015;36:513-23.

109. Dewan S, McCabe K, Regnier M, et al. Molecular effects of cardiac troponin DCM mutations on calcium sensitivity and Myofilament activation - an integrated multi-scale modeling study. Biophys J 2017;112:322a.

110. Elmore DE, Dougherty DA. Molecular dynamics simulations of wild-type and mutant forms of the mycobacterium tuberculosis MscL channel. Biophys J 2001;81:1345-59.

111. Feng $Z$, Alqarni $M H$, Yang $P$, et al. Modeling, molecular dynamics simulation, and mutation validation for structure of cannabinoid receptor 2 based on known crystal structures of GPCRs. J Chem Inf Model 2014;54: 2483-99.

112. Frappier V, Chartier M, Najmanovich RF. ENCoM server: exploring protein conformational space and the effect of mutations on protein function and stability. Nucleic Acids Res 2015;43:W395-400.

113. Gapsys V, Michielssens S, Seeliger D, et al. Accurate and rigorous prediction of the changes in protein free energies in a large-scale mutation scan. Angew Chem Int Ed 2016; 55:7364-8.

114. Jordan EJ, Radhakrishnan R. We can predict the effects of kinase domain mutations using molecular dynamics and machine learning. Biophys J 2017;112:322a.

115. Koukos PI, Glykos NM. Folding molecular dynamics simulations accurately predict the effect of mutations on the stability and structure of a vammin-derived peptide. J Phys Chem 2014;118:10076-84.

116. Kumar A, Purohit R. Use of long term molecular dynamics simulation in predicting cancer associated SNPs. PLoS Comput Biol 2014;10:e1003318.

117. Padhi AK, Vasaikar SV, Jayaram B, et al. ANGDelMut - a web-based tool for predicting and analyzing functional loss 
mechanisms of deleterious angiogenin mutations causing amyotrophic lateral sclerosis. F1000Res 2013;2.

118. Rodrigues CHM, Pires DEV, Ascher DB. DynaMut: predicting the impact of mutations on protein conformation, flexibility and stability. Nucleic Acids Res 2018; gky300.

119. Schadzek P, Schlingmann B, Schaarschmidt F, et al. Data of the molecular dynamics simulations of mutations in the human connexin46 docking interface. Data Brief 2016;7:93-9.

120. Zimmermann MT, Urrutia R, Oliver GR, et al. Molecular modeling and molecular dynamic simulation of the effects of variants in the TGFBR2 kinase domain as a paradigm for interpretation of variants obtained by next generation sequencing. PLos One 2017;12:e0170822.

121. Hensen $U$, Meyer $T$, Haas J, et al. Exploring protein dynamics space: the dynasome as the missing link between protein structure and function. PLos One 2012;7:e33931.

122. Henzler-Wildman $\mathrm{KA}$, Thai $\mathrm{V}$, Lei $\mathrm{M}$, et al. Intrinsic motions along an enzymatic reaction trajectory. Nature 2007;450:838-44.

123. Stein A, Rueda M, Panjkovich A, et al. A systematic study of the energetics involved in structural changes upon association and connectivity in protein-protein interaction networks. Structure 2011;19:881-9.

124. Gerstein M, Krebs W. A database of macromolecular motions. Nucleic Acids Res 1998;26:4280-90.

125. Orozco M. The dynamic view of proteins: comment on "comparing proteins to their internal dynamics: exploring structure-function relationships beyond static structural alignments". Phys Life Rev 2013;10:29-30.

126. Case DA. Normal mode analysis of protein dynamics. Curr Opin Struct Biol 1994;4:285-90.

127. Pires DEV, Ascher DB, Blundell TL. mCSM: predicting the effects of mutations in proteins using graph-based signatures. Bioinformatics 2014;30:335-42.

128. Capriotti E, Fariselli P, Casadio R. I-Mutant2.0: predicting stability changes upon mutation from the protein sequence or structure. Nucleic Acids Res 2005;33:W306-10.

129. Pires DEV, Ascher DB, Blundell TL. DUET: a server for predicting effects of mutations on protein stability using an integrated computational approach. Nucleic Acids Res 2014;42:W314-9.

130. Pandurangan AP, Ochoa-Montaño B, Ascher DB, et al. SDM: a server for predicting effects of mutations on protein stability. Nucleic Acids Res 2017;45:W229-35.

131. Schymkowitz J, Borg J, Stricher F, et al. The FoldX web server: an online force field. Nucleic Acids Res 2005;33:W382-8.

132. Dehouck Y, Grosfils A, Folch B, et al. Fast and accurate predictions of protein stability changes upon mutations using statistical potentials and neural networks: PoPMuSiC-2.0. Bioinformatics 2009;25:2537-43.

133. Pucci F, Bernaerts KV, Kwasigroch JM, et al. Quantification of biases in predictions of protein stability changes upon mutations. Bioinformatics 2018;34:3659-65.

134. Masso M, Vaisman I. AUTO-MUTE: web-based tools for predicting stability changes in proteins due to single amino acid replacements. Protein Eng Des Sel 2010;23:683-7.

135. Masso M, Vaisman II. AUTO-MUTE 2.0: a portable framework with enhanced capabilities for predicting protein functional consequences upon mutation. Advances in bioinformatics 2014;2014:278385-5.

136. Hospital A, Goñi JR, Orozco M, et al. Molecular dynamics simulations: advances and applications. Adv Appl Bioinform Chem 2015;8:37-47.
137. Kumari I, Sandhu P, Ahmed M, et al. Molecular dynamics simulations, challenges and opportunities: a Biologist's prospective. Curr Protein Pept Sci 2017;18:1163-79.

138. Galano-Frutos JJ, Sancho J. Accurate calculation of Barnase and SNase folding energetics using short MD simulations and an atomistic model of the unfolded ensemble. Evaluation of force fields and water models. J Chem Inf Model 2019In Press. doi: 10.1021/acs.jcim.9b00430.

139. Nerenberg PS, Head-Gordon T. New developments in force fields for biomolecular simulations. Curr Opin Struct Biol 2018;49:129-38.

140. Ouyang JF, Bettens RP. Modelling water: a lifetime enigma. Chimia 2015;69:104-11.

141. Martínez-Oliván J, Arias-Moreno X, Velazquez-Campoy A, et al. LDL receptor/lipoprotein recognition: endosomal weakening of $A p o B$ and ApoE binding to the convex face of the LR5 repeat. FEBS J 2014;281:1534-46.

142. Rudenko G, Henry L, Henderson K, et al. Structure of the $\mathrm{LDL}$ receptor extracellular domain at endosomal $\mathrm{pH}$. Science 2002;298:2353-8.

143. García-Fandiño R, Bernadó P, Ayuso-Tejedor S, et al. Defining the nature of thermal intermediate in 3 state folding proteins: apoflavodoxin, a study case. PLoS Comput Biol 2012;8:e1002647.

144. Micheletti C. Comparing proteins by their internal dynamics: exploring structure-function relationships beyond static structural alignments. Phys Life Rev 2013;10:1-26.

145. Velázquez-Muriel RM, Cuesta I, et al. Comparison of molecular dynamics and superfamily spaces of protein domain deformation. BMC Struct Biol 2009;9:6.

146. Ghosh T, García AE, Garde S. Molecular dynamics simulations of pressure effects on hydrophobic interactions. J Am Chem Soc 2001;123:10997-1003.

147. Okumura $\mathrm{H}$. Temperature and pressure denaturation of chignolin: folding and unfolding simulation by multibaricmultithermal molecular dynamics method. Proteins 2012;80:2397-416.

148. Day R, Bennion BJ, Ham S, et al. Increasing temperature accelerates protein unfolding without changing the pathway of unfolding. J Mol Biol 2002;322:189-203.

149. Bennion BJ, Daggett V. The molecular basis for the chemical denaturation of proteins by urea. Proc Natl Acad Sci U S A 2003;100:5142-7.

150. Camilloni C, Guerini Rocco A, Eberini I, et al. Urea and guanidinium chloride denature protein $\mathrm{L}$ in different ways in molecular dynamics simulations. Biophys J 2008;94:4654-61.

151. Gao M, Wilmanns M, Schulten K. Steered molecular dynamics studies of Titin I1 domain unfolding. Biophys $J$ 2002;83:3435-45.

152. Lu H, Schulten $\mathrm{K}$. Steered molecular dynamics simulations of force-induced protein domain unfolding. Proteins 1999; 35:453-63.

153. Krieger E, Vriend G. New ways to boost molecular dynamics simulations. J Comput Chem 2015;36:996-1007.

154. Biedermann J, Ullrich A, Schöneberg J, et al. ReaDDyMM: fast interacting particle reaction-diffusion simulations using graphical processing units. Biophys J 2015;108:457-61.

155. Barney L. Speeding up molecular dynamics: modified GROMACS code improves optimization, parallelization. Scientific Computing Advantage Business Marketing 2016.

156. Shaw DE, Dror RO, Salmon JK, et al. Millisecond-scale molecular dynamics simulations on Anton. In: SC'09: Proceedings of the Conference on High Performance Computing Networking, Storage and Analysis. New York, NY, 2009, 1-11. 
157. Kleinjung J, Fraternali F. Design and application of implicit solvent models in biomolecular simulations. 2014;25, 100 : 126-34.

158. Nielsen SO, Bulo RE, Moore PB, et al. Recent progress in adaptive multiscale molecular dynamics simulations of soft matter. Phys Chem Chem Phys 2010;12:12401-14.

159. Pronk S, Larsson P, Pouya I, et al. Copernicus: a new paradigm for parallel adaptive molecular dynamics. In: SC '11: Proceedings of 2011 International Conference for High Performance Computing, Networking, Storage and Analysis. New York, NY: ACM, 2011.

160. Riniker S, van Gunsteren WF. Mixing coarse-grained and fine-grained water in molecular dynamics simulations of a single system. J Chem Phys 2012;137:044120.

161. Freddolino PL, Harrison CB, Liu Y, et al. Challenges in protein folding simulations: timescale, representation, and analysis. Nat Phys 2010;6:751-8.

162. Lindahl E, Hess B, van der Spoel D. GROMACS 3.0: a package for molecular simulation and trajectory analysis. J Mol Model 2001;7:306.

163. Michaud-Agrawal N, Denning EJ, Woolf TB, et al. MDAnalysis: a toolkit for the analysis of molecular dynamics simulations. J Comput Chem 2011;32:2319-27.

164. Roe DR, Cheatham IIITE. PTRAJ and CPPTRAJ: software for processing and analysis of molecular dynamics trajectory data. J Chem Theory Comput 2013;9:3084-95.

165. Papaleo E, Saladino G, Lambrughi $M$, et al. The role of protein loops and linkers in conformational dynamics and allostery. Chem Rev 2016;116:6391-423.

166. Kryshtafovych A, Monastyrskyy B, Fidelis K, et al. Evaluation of the template-based modeling in CASP12. Proteins 2018;86:321-4.

167. Smith LM, Kelleher NL. Proteoform: a single term describing protein complexity. Nat Methods 2013;10:186-7.

168. Manavalan B, Kuwajima K, Lee J. PFDB: a standardized protein folding database with temperature correction. Sci Rep 2019;9:1588.

169. Zeldovich KB, Chen P, Shakhnovich EI. Protein stability imposes limits on organism complexity and speed of molecular evolution. Proc Natl Acad Sci USA 2007;104: 16152-7.

170. Tokuriki N, Tawfik DS. Stability effects of mutations and protein evolvability. Curr Opin Struct Biol 2009;19: 596-604.

171. Campos LA, Garcia-Mira MM, Godoy-Ruiz R, et al. Do proteins always benefit from a stability increase? Relevant and residual stabilisation in a three-state protein by charge optimisation. J Mol Biol 2004;344:223-37.

172. Moore GE. Progress in digital integrated electronics [Technical literature, Copyright 1975 IEEE. Reprinted, with permission. Technical Digest. International Electron Devices Meeting, IEEE, 1975, pp. 11-13]. IEEE Solid-State Circuits Society Newsletter 2006;11:36-7.

173. Bilsel O, Matthews CR. Barriers in protein folding reactions. Adv Protein Chem 2000;53:153-207.

174. Preeprem T, Gibson G. SDS, a structural disruption score for assessment of missense variant deleteriousness. Front Genet 2014;5:82.

175. Tanyalcin I, Stouffs K, Daneels D, et al. Convert your favorite protein modeling program into a mutation predictor: "MODICT". BMC Bioinformatics 2016;17:425-5.

176. Pires DEV, Ascher DB. mCSM-NA: predicting the effects of mutations on protein-nucleic acids interactions. Nucleic Acids Res 2017;45:W241-6
177. Calabrese R, Capriotti E, Fariselli P, et al. Functional annotations improve the predictive score of human diseaserelated mutations in proteins. Hum Mutat 2009;30:1237-44.

178. Capriotti E, Calabrese R, Fariselli P, et al. WS-SNPs\&GO: a web server for predicting the deleterious effect of human protein variants using functional annotation. BMC Genomics 2013;14:S6

179. Mi H, Huang X, Muruganujan A, et al. PANTHER version 11: expanded annotation data from Gene Ontology and Reactome pathways, and data analysis tool enhancements. Nucleic Acids Res 2017;45:D183-9.

180. Stone EA, Sidow A. Physicochemical constraint violation by missense substitutions mediates impairment of protein function and disease severity. Genome Res 2005;15: 978-86.

181. Capriotti E, Calabrese R, Casadio R. Predicting the insurgence of human genetic diseases associated to single point protein mutations with support vector machines and evolutionary information. Bioinformatics 2006;22:2729-34.

182. Tavtigian SV, Deffenbaugh AM, Yin L, et al. Comprehensive statistical study of 452 BRCA1 missense substitutions with classification of eight recurrent substitutions as neutral. J Med Genet 2006;43:295-305.

183. Tian J, Wu N, Guo X, et al. Predicting the phenotypic effects of non-synonymous single nucleotide polymorphisms based on support vector machines. BMC Bioinformatics 2007;8:450.

184. Carter H, Chen S, Isik L, et al. Cancer-specific highthroughput annotation of somatic mutations: computational prediction of driver missense mutations. Cancer Res 2009;69:6660-7.

185. Schwarz JM, Cooper DN, Schuelke M, et al. MutationTaster2: mutation prediction for the deep-sequencing age. Nat Methods 2014;11:361-2.

186. Schwarz JM, Rödelsperger C, Schuelke M, et al. MutationTaster evaluates disease-causing potential of sequence alterations. Nat Methods 2010;7:575-6.

187. Reva B, Antipin Y, Sander C. Predicting the functional impact of protein mutations: application to cancer genomics. Nucleic Acids Res 2011;39:e118.

188. Choi Y. A fast computation of pairwise sequence alignment scores between a protein and a set of single-locus variants of another protein. In: Proceedings of the ACM Conference on Bioinformatics, Computational Biology and Biomedicine (BCB '12). New York, NY, 2012, 414-7.

189. Choi Y, Sims GE, Murphy S, et al. Predicting the functional effect of amino acid substitutions and indels. PLos One 2012;7:e46688.

190. Makarov V, O'Grady T, Cai G, et al. AnnTools: a comprehensive and versatile annotation toolkit for genomic variants. Bioinformatics 2012;28:724-5.

191. Shihab HA, Gough J, Cooper DN, et al. Predicting the functional, molecular, and phenotypic consequences of amino acid substitutions using hidden Markov models. Hum Mutat 2013;34:57-65.

192. Katsonis P, Lichtarge O. A formal perturbation equation between genotype and phenotype determines the evolutionary action of protein-coding variations on fitness. Genome Res 2014;24:2050-8.

193. Niroula A, Urolagin S, Vihinen M. PON-P2: prediction method for fast and reliable identification of harmful variants. PLos One 2015;10:e0117380.

194. Karchin R, Diekhans M, Kelly L, et al. LS-SNP: largescale annotation of coding non-synonymous SNPs based 
on multiple information sources. Bioinformatics 2005;21: 2814-20.

195. Ryan M, Diekhans M, Lien S, et al. LS-SNP/PDB: annotated non-synonymous SNPs mapped to Protein Data Bank structures. Bioinformatics 2009;25:1431-2.

196. Ferrer-Costa C, Parraga I, Gelpí JL, et al. PMUT: a web-based tool for the annotation of pathological mutations on proteins. Bioinformatics 2005;21:3176-8.

197. López-Ferrando V, Gazzo A, de la Cruz X, et al. PMut: a webbased tool for the annotation of pathological variants on proteins, 2017 update. Nucleic Acids Res 2017;45:W222-8.

198. Yue P, Melamud E, Moult J. SNPs3D: candidate gene and SNP selection for association studies. BMC Bioinformatics 2006;7:166.
199. Hecht M, Bromberg Y, Rost B. Better prediction of functional effects for sequence variants. BMC Genomics 2015;16:S1.

200. Capriotti E, Fariselli P, Rossi I, et al. A three-state prediction of single point mutations on protein stability changes. BMC Bioinformatics 2008;9:S6.

201. Capriotti E, Altman RB. Improving the prediction of diseaserelated variants using protein three-dimensional structure. BMC Bioinformatics 2011;12(S3).

202. Acharya V, Nagarajaram HA. Response to: statistical analysis of missense mutation classifiers. Hum Mutat 2013; 34:407.

203. Pei J, Grishin NV. AL2CO: calculation of positional conservation in a protein sequence alignment. Bioinformatics 2001;17:700-12. 